\title{
A SIMULATION FRAMEWORK FOR REAL-TIME ASSESSMENT OF DYNAMIC RIDE SHARING DEMAND RESPONSIVE TRANSPORTATION MODELS
}

\author{
M.Paz Linares \\ Lídia Montero \\ Jaume Barceló \\ Department of Statistics and Operations Research \\ and inLab FIB \\ Universitat Politècnica de Catalunya-UPC \\ Carrer Jordi Girona 1-3 \\ 08034-Barcelona, SPAIN
}

\author{
Carlos Carmona
}

inLab FIB

Universitat Politècnica de Catalunya-UPC

Carrer Jordi Girona 1-3

08034-Barcelona, SPAIN

\begin{abstract}
Sustainable mobility is not only a technological question, automotive technology will be part of the solution combined with a paradigm shift from car ownership to vehicle usage, and the application of Information and Communication Technologies (ICT) making possible for a user to have access to a mobility service from anywhere to anywhere at any time. Multiple Passenger Ridesharing and its variants look one of the more promising emerging mobility concepts. However, implementations of these systems accounting specifically for time dependencies, and time windows reflecting users' needs raise challenges in terms of real-time fleet dispatching and dynamic route calculation. This paper analyzes and evaluates both aspects by microscopic traffic simulation emulating real-time traffic conditions and a real traffic information system, and interacting with a Decision Support. The simulation framework has been implemented in a model of Barcelona's Central Business District. The paper presents and discusses the achieved results.
\end{abstract}

\section{INTRODUCTION}

Urban areas must address from a holistic perspective the challenges and threads of sustainability namely in providing services to companies and citizens. Cities are complex systems, any city must be thought as a "System of Systems", and Mobility is only one of the components of such complex systems, a nonisolated component strongly interacting with all other components and therefore its implications must be analyzed in the context of these interactions. Frost \& Sullivan, in a recent analysis of the Future of Mobility and New Mobility Business Models, (Sullivant \& Frost 2015) identify the growing trend of "Ride Sharing" models as one of what they call "Transformational Shifts in Mobility". This trend can be seen as one of the consequences of the paradigmatic shift from "car ownership" to "vehicle usage", leading to a new concept of multi modal mobility network, as a way of overcoming the limitations of conventional public transport systems, made possible by the pervasive penetration of ICT. Technology enables a comfortable seamless real-time point to point travel service.

Demand Responsive Transport (DRT), Dial-a-Ride Transit or Flexible Transport Services, according with the definition of the European Commission, Directorate-General for Energy and Transport "are emerging user-oriented forms of public transport characterized by flexible routing and scheduling of small/medium vehicles operating in shared-ride mode between pick-up and drop-off locations according 


\section{Linares, Montero, Barceló and Carmona}

to passenger needs". DRT were initially thought to provide public transport services for areas with low passenger demand where regular bus services would not be available. However, this concept is quickly evolving enabled by the ICT deployment, initiatives like KUTSUPLUS (Anon 2015), an on-demand minibus service run by Helsinki's public transit authority, letting riders choose their own route, summon a trip with a smartphone, decide the start and end point of their trip and choose whether to share a journey or not. It is a new Demand Responsive Public Transport service designed to achieve maximum flexibility.

This concept of Demand Responsive Transport is rapidly evolving to services provided by private companies operating point to point with full dynamics and flexibility, also offering the possibility of sharing trips. E-hailing is a process of ordering a transportation service by a private car (e.g. Uber services), special taxi services, etc. The system has currently a variety of implementations but, in essence, the variant we are interested in this paper, assumes that the customer books or hails the trip electronically providing the pickup location (that can be automatically identified by GPS current customer location), the drop off location, and the desired pickup up and drop off time windows, and that multiple passengers can share the trip. In order words our research addresses specifically the variant known a "Multiple Passengers Ridesharing System" (MPRS).

A state-of-the art-survey on the variants of ridesharing systems, their alternatives and likely future evolution can be found in (Furuhata et al. 2013), according to their classification the variant studied in this paper corresponds to the called Detour Ridesharing with Multiple Passengers, in which multiple passengers, with relatively close but different origins and destinations share rides which can partial or totally overlap. Our research has analyzed the potential utilization uses of special fleets of dedicated vehicles in an urban area, assuming than further than the pickup and drop off location and time windows, the system is also aware of the current and desirably short term forecasted traffic conditions, supplied by an Advanced Traveler Information System (ATIS), to timely determine the optimal routes satisfying customers' time constraints. This type of real-time ridesharing system has also been studied by (Ma et al. 2015) but with simplifications concerning the availability of traffic information. The special case when the fleet of service vehicles is composed of autonomous vehicles has deserved special attention from the agent-based simulation approach, a general perspective can be found in (Fagnant \& Kockelman 2014) that analyze the environmental impacts, while (Martinez et al. 2015) propose a general agent-based simulation to assess the impacts, and apply it to the city of Lisbon. This work has been the basis for the report of the International Transport Forum (OECD - International Transport Forum 2015). One of critical aspects highlighted in these last references concerns the operational efficiency of the system, determined, among other factors by the fleet management dispatching system, in terms of the decisions process and its dependencies with the size of the fleet and the demand, an aspect that has been studied in (Boesch et al. 2016) for a simplified dispatching strategy. Consequently our work has integrated the Decision Support System (DSS) supporting the decision making process of which vehicle assign to optimally provide the requested service for a customer, taking into account the new service requirements, the confirmed rights of the customers already being provided a service, and the current and estimated travel times in the urban network provided by a microscopic traffic simulation platform. The system whose evaluation is object of this paper is assumed to work under the following modelling hypothesis:

1. There is a fleet of $n$ dedicated identical vehicles with a capacity for $p$ passengers each. The system is continuously tracking the fleet vehicles and a t each instant in time knows its current position, space availability, destinations and expected arrival times of customer being serviced. These vehicles can be in various states:

a. Idle, either traveling in the road network, or parked at a designated location, waiting for a service request.

b. In service, but not at full capacity, providing service to various customers.

c. In service but full, and therefore unable to provide a new service.

2. At a given time $t_{i}$ a client (let's assume the $i$-th client) $C_{i}$, calls for a service. The system 


\section{Linares, Montero, Barceló and Carmona}

automatically identifies the client's location (e.g. by georeferencing) and the user informs on his/her destination $D_{i}$, the time window ( $e_{i}$, earliest time, $l_{i}$, latest time) for his/her pick up expectancy (how long accepts him/she waiting to be pick up), and the desired arrival time to his/her destination, that is an expected arrival time $a_{i}$ and a time slack $\varepsilon_{i}$.

3. When the system receives the new service request checks the status of the vehicle fleet to determine whether any of the vehicles, currently in service or idle, can feasibly provide the new service making a detour, if necessary, to collect the new customer, to satisfy his/her conditions without violating the requirements of the customers currently on board. That is, the system checks the feasibility of the new service.

4. In case there is more than a vehicle available for that service a Decision Support System determines which is the most profitable, both from the customer's point of view in terms of the quality of the system and from the system's point of view looking for the cheaper service.

5. Then the system assigns the service to the selected vehicle and the most efficient route to do it, including potential detours and changes in the previous routes.

6. To achieve the highest potential added value the system assumes to have access to an ATIS, providing current and short term forecasted link travel times on all traffic network links.

To conduct the analysis and evaluation of the proposed MPRS by simulation the simulator to be used:

1. Must be able of representing the road network geometry in full detail, i.e. importing it as it is from a Geographic Information System, including the explicit representations of traffic control strategies at signalized intersections

2. It must be able of realistically emulate the time evolution of traffic flows on the road network by properly describing the dynamics of individual vehicles on basis to model driving behavior in terms of leader-follower, lane changing, gap acceptance, etc. models. Including stochastic parameters for appropriately mimicking human driving behavior.

3. It must allow dealing with multiple vehicle classes with differentiated behaviors, in order to be able of accounting for the specific characteristic of the vehicles of the fleets object of study

4. Vehicles in the simulation model travel from origins to destinations along time dependent routes selected according to stochastic route choice models that are timely updated (e.g. as in the case of the rerouting of the fleet vehicles to provide new services)

5. The simulation platform must have the way of interacting with external applications, as those implementing the functions of the Decision Support System.

In summary these requirements mean that the appropriate simulator is a microscopic traffic simulator with the required extended functionalities. The proposed system has been tested by microscopic traffic simulation using a model of the Barcelona's Business District, defining specialized fleets and using as design factors in the simulation experiments the demand of shared vehicles, the sizes $\mathrm{p}$ of the shared vehicles, the size $\mathrm{n}$ of the fleet providing the service, the conditions on the customers' time windows (e.g. tight or soft) and the degree of dynamism, that is the \% with respect to the total demand of the ridesharing services that is not known before the start of the journey.

\section{SYSTEM'S FRAMEWORK AND ARCHITECTURE}

The ¡Error! No se encuentra el origen de la referencia. Figure 1 depicts the system's architecture that implements a simulation framework to test the feasibility and performance of the MPRS, which combines a microscopic traffic simulation with a Decision Support Module which includes the Dynamic Router and Scheduler (DRS). The microscopic traffic simulator emulates with great detail and realism the traffic conditions on a road network, as well as the information provided by ICT (for instance, by GPS), supplying all information needed during the process, and performing the other tasks required along the 


\section{Linares, Montero, Barceló and Carmona}

global process. The DRS lays at the core of the framework and consists on a set of algorithms providing on-line solution to the real-time vehicle routing problem with time windows and time dependencies. The DRS is triggered every time an event, external or internal, is detected by the system. The external events depend on the demand, while the internal events, depend on the observed traffic flow dynamics which could be the cause of delays in the estimated arrival times to the location of customers. Events randomly occurring during the fleet operations are generated by the microscopic traffic simulator according to stochastic patterns.

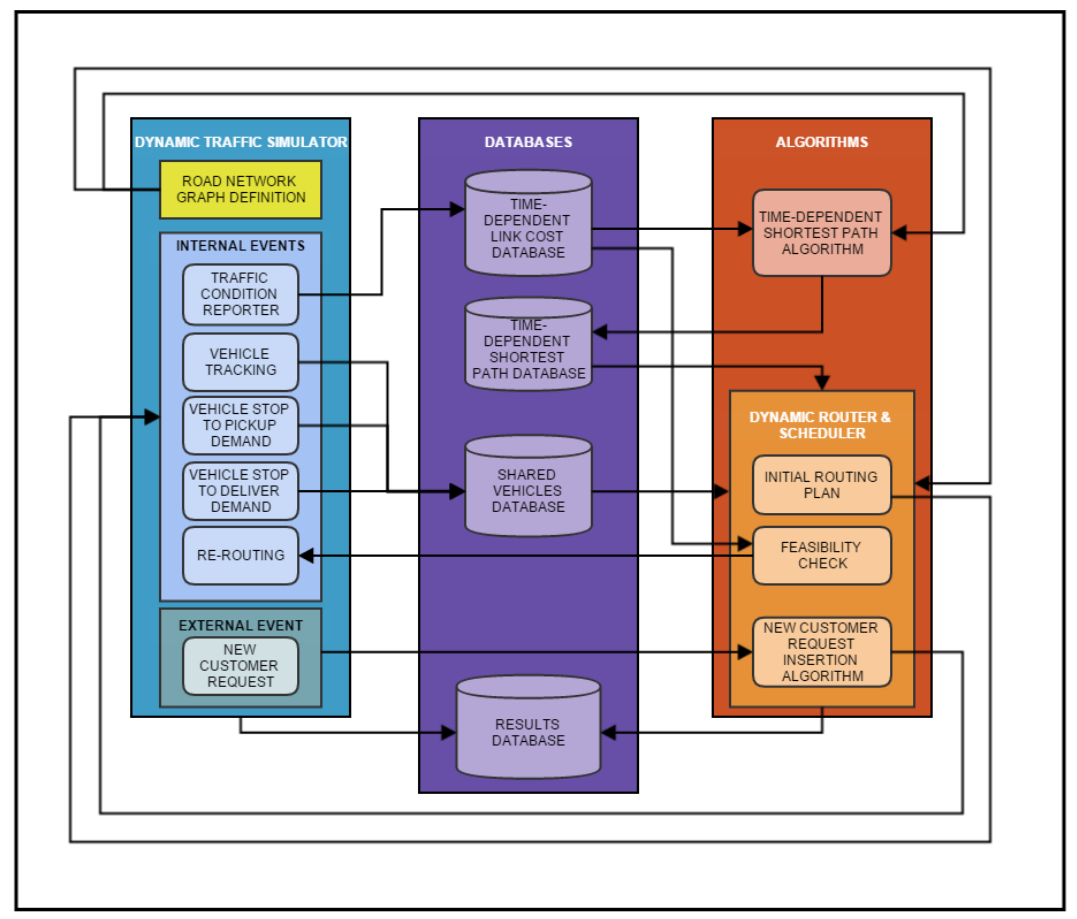

Figure 1: System's Architecture

\subsection{Algorithms}

It hosts the different algorithms needed to provide an on-line solution to the real-time vehicle routing problem.

\subsubsection{Dynamic Router \& Scheduler}

As core of the computational framework the DRS consists on a set of algorithmic tools providing on-line solutions to the proposed real-time vehicle routing problems. The algorithm used can be exact algorithms, heuristics or hybrid approaches.

In the case analyzed in this paper we focus on the use of dynamic insertion heuristic solutions based on feasibility and profitability concepts. Feasibility is considered in the sense that vehicles serving those routes have enough time to travel to the new customer taking into account the time windows constraints of all customers already being served. The profit of a new insertion is the negative of the additional travel time incurred from inserting the new customer in a route. Its required inputs are:

- The time-dependent link costs, stored into the Time-dependent Link Cost DB.

- The time-dependent shortest paths, stored into the Time-dependent Shortest Path DB. 


\section{Linares, Montero, Barceló and Carmona}

- The cooperative vehicle fleet state reported by the Dynamic Traffic Simulator and stored into the Shared Vehicles DB.

- The road network graph. This graph, stored in a file into the server, will be generated offline during the installation phase.

It contains three modules in order to respond to three different problems:

- Initial Routing Plan: To find the initial routing plan. In a first step, the initial routing plan is computed considering the pre-booked demand that must be served at the beginning of the journey.

- New Customer Request Insertion Algorithm: To give an answer to new costumers requests, assigning a vehicle to pick-up this customer and a route to reach the customer's destination. When a new customer request arrives this module fires an execution, and the vehicle routing problem is solved. When a solution is reached, this module generates a response to the simulator component divided in two parts. On one hand, it informs the customer accepting its requests and giving him information about the vehicle assigned. On the other hand, the assigned vehicle receives a new route in order to pick up its new passenger.

- Feasibility Check: To check if the previously assigned routes are feasible after a traffic condition update, recalculating the routes when needed. Feasibility is considered in the sense that vehicles serving those routes have enough time to travel to its passengers' destinations. This module checks if all the currently assigned routes are still feasible with the new traffic condition. If the current solution is not feasible, new routes will be calculated and cooperative vehicles affected by the routes changes will be informed with the new routes.

\subsubsection{Time-Dependent Shortest Path Algorithm}

It is the responsible of the Time-Dependent Shortest Path (TDSP) calculation. All the routes and its travel times from any section to any section of the network will be calculated and stored into the TDSP DB. Its required inputs are: the link travel costs provided by the Traffic Condition Reporter, stored in the Links Travel Cost DB; and the network graph, stored in a file into the server, will be generated offline during the installation phase.

The algorithm calculates the optimal vehicle paths among the pickup and delivery stops and the corresponding travel time. The shortest path problem considered in our research differs from the usual shortest path approaches in that we don't considers link travel times constant but time dependent, in other words, the link cost depends on the arrival time at the origin of the link, that is the considered shortest paths are dynamic or time-dependent (TDSP).

In this work, we have implemented a variation of the DOT (Decreasing Order of Time) TDSP algorithm proposed by (Barceló et al. 2013). The authors present an implementation of the DOT TDSP by (Chabini 1998) using the structures proposed by (Ziliaskopoulos \& Mahmassani 1993). The main features of this solution are the reduction of the time achieved using the DOT concept and the reduction of inmemory space by using the Yale format for Sparse Matrices. A detailed description of the algorithm can be found in the reference (Barceló et al. 2013).

\subsection{Microscopic Traffic Simulator}

The second component of the computational framework is a microscopic traffic simulator, with the functionalities already described in the Introduction Section, which additionally: generates databases of time-dependent link travel times; randomly generates the different external and internal events previously mentioned; tracks fleet vehicle positions and state at every time step of the simulation; assigns dynamic routes to fleet vehicles; provides to the DRS the input data required by the algorithms.

In this work we selected Aimsun v7 (Transport Simulation Systems 2013) among the currently existing microscopic traffic simulators because its functional architecture, and the Micro API (APPI) auxiliary 


\section{Linares, Montero, Barceló and Carmona}

tool, supports the required extended modeling utilities, for working with the user applications, that implement the algorithms required for the study.

The exchange of information between the external application and the microsimulator is done at every simulation step, which is the time interval at which the estate of the simulation model is updated. The development languages have been $\mathrm{C}++$ and Python.

The key system components are: the Road Network Definition; the Internal Events (Traffic Condition Reporter, Vehicle Tracking, Vehicle Stop to Pick up Demand, Vehicle Stop to Deliver Demand and Rerouting); and the External Event (New customer Request).

\subsection{Databases}

The information generated by the simulator and the applications modules is stored in a set of Databases:

- Time-Dependent Link Cost Database: To store the link travel cost provided by the Traffic Condition Reporter. TDSP Database: To store all the generated routes with it travel time from any section to any section of the network.

- Shared Vehicles Database: To maintain updated information of the vehicle fleet state. It stores the position (UTM coordinates) and state (customer pickup, arrival to destination) of every fleet vehicle.

- Results Database: To store the results achieved in every performed simulation and the calculated Key Performance Indicators.

The flow chart shown in Figure 2 summarizes the described process. When a simulation starts, it communicates its corresponding settings to the server. These settings are stored into a Redis database and the TDSP algorithm starts in a new thread. In parallel, the Vehicle Routing Problem (VRP) Algorithm is triggered and the Pre-booked Demand begins to be loaded. The VRP waits for two synchronization points: the TDSP needs to be computed and the Pre-booked Demand load must end. Then, the VRP creates the initial solution. From this point the server keeps attending the demand requests as they arrive until the end of the simulation.

\section{REAL-TIME TRAFFIC INFORMATION SYSTEM}

The described computational framework to test the MPRS assumes that the fleet management approach and the core component of the DRS are embedded into a microscopic traffic simulation model in terms of a process whose logic is the following:

1. Selection of a time interval step to check the feasibility of the current routes.

2. At each time interval step, the simulator sends to the link costs data base the current travel times of each link of the network.

3. With this new information, the TDSP module re-computes the TDSP between all the nodes of the network at each departure time interval.

4. With these new TDSP costs, the TDSP data base is updated.

i. The feasibility check method is launched using this new information stored in the TDSP DB.

ii. Modifies the current solution to solve the feasibility problems.

The microscopic traffic simulation plays a twofold role; the exchange of information required by the logics of the decision made by the fleet dispatcher and the real-time information, including link travel times, required by the dynamic routing algorithms. In other words, in a real life system we would consider that the fleet manager has access to an ATIS supplying such information. In the computational implementation of the system we have considered that the emulation of such ATIS was also the function of the dynamic traffic simulator. Therefore the fleet manager has access to such information and the vehicle routing algorithms are able of "continuously" checking the traffic conditions as well as the fleet expected timing. In this way, the algorithm is able to manage sudden changes in real traffic travel times. 
Linares, Montero, Barceló and Carmona

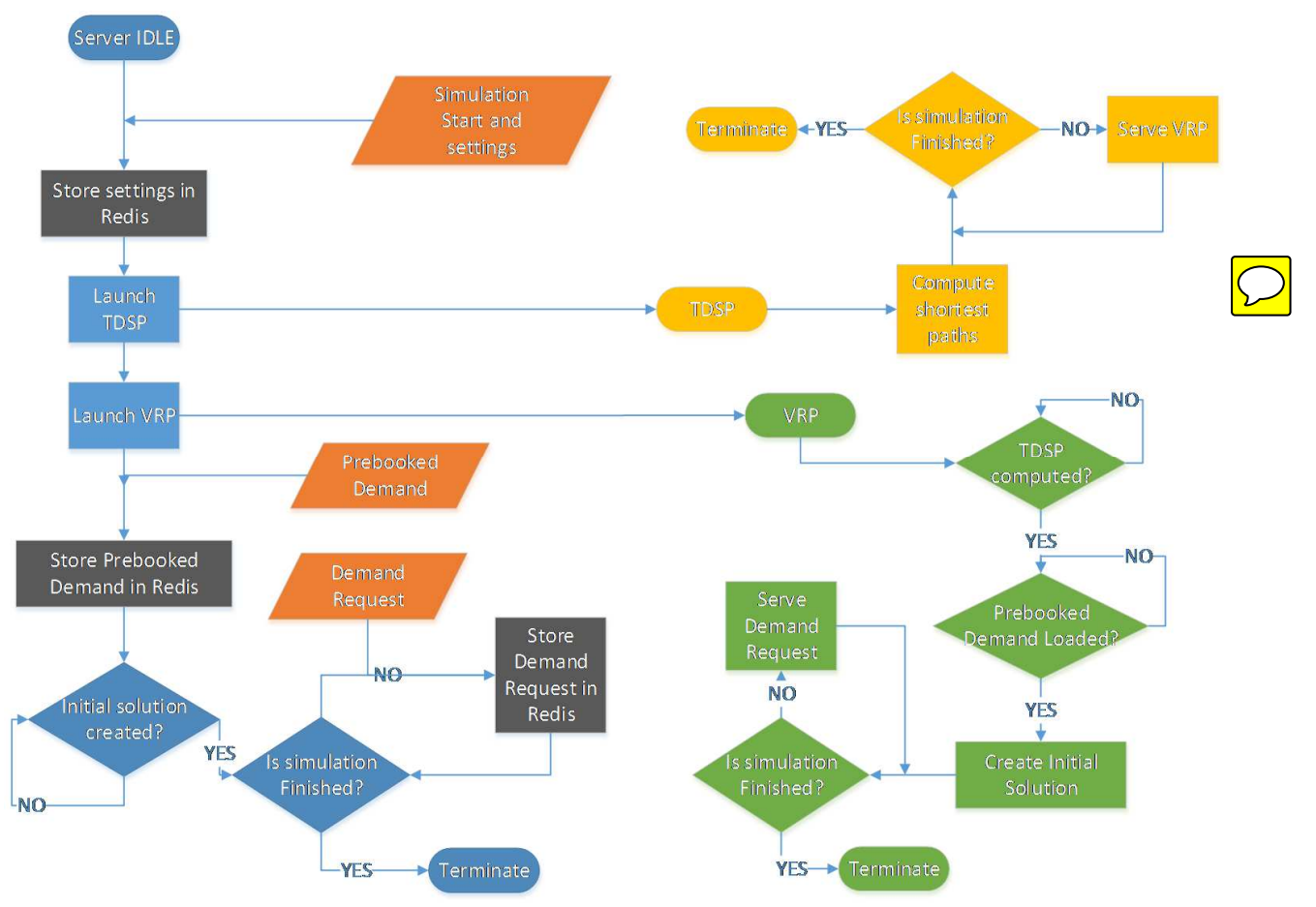

Figure 2: Flow Diagram of the system process

\section{DESIGN OF EXPERIMENTS}

\subsection{Model Description}

The selected microsimulation scenario was Barcelona's CBD, the "Eixample" (see Figure 3), comprising $7.46 \mathrm{~km} 2$ with more than 250,000 inhabitants. Its Aimsun (Transport Simulation Systems 2013) model consists of 1720 links, 528 nodes, 120/130 generation and attraction centroids and 877 nonzero OD pairs. The horizon study is $30 \mathrm{~min}$, accounting for a total of 20,700 vehicle trips. The microscopic traffic simulator model updates the model state every half a second, updating positions of all vehicle classes considered: passenger cars, buses and ride-sharing vehicles.
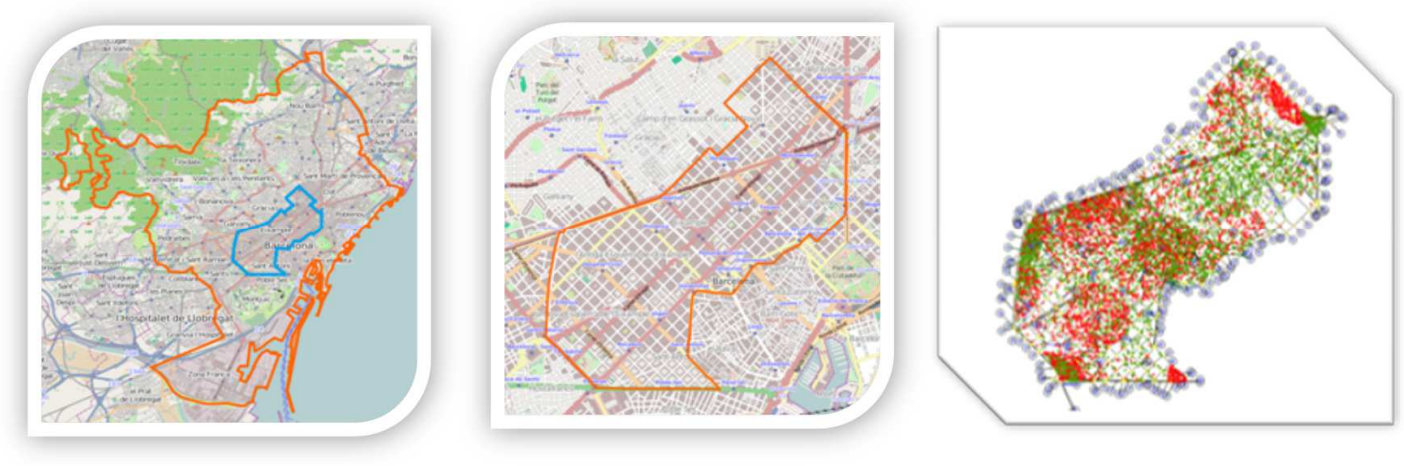

Figure 3: L'Eixample in Barcelona (left) - simulation test-site (central) and Aimsun model (right) with spatial distribution of demand requests for the new mode in Base scenario. 


\section{Linares, Montero, Barceló and Carmona}

Passenger cars demand is modelled as 15-min time-sliced demand whose Origin-Destination pattern reproduces the actual working day morning period in Eixample district. The model includes the detailed description of the 50 routes operating in the "Eixample" accounting explicitly for frequencies and stops for boarding/alighting and finally, a fleet of multiple passenger ride-sharing units (MPRS units) that are moved over the network according to the dynamic routing and scheduling to serve passenger requests. The fleet of shared units dispatching considers time-dependent routing algorithms according to real-time emulated traffic conditions provided by the simulator.

\subsection{Methodological Proposal}

The goal was to evaluate the new transport mode in a urban area with regard to the impacts on urban traffic and taking into account a very detailed description of the characteristics of the mobility service. The implementation of mobility strategies into a specific real scenario was considered. The system was designed to be flexible to host a wide range of service possibilities by setting the values of parameters determining the behavior of the system like :

- Fleet size, vehicle capacity the market quota for the new mode as a percentage of the total passengers cars demand for the study period,

- Fully flexible routes, fixed routes or mixed routing

- Boarding/Alighting allowed either everywhere in the network or at pre-defined stops or regular-bus stops or any chamfer of Eixample geometry

- Depot existence: number and location

- Pre-booked demand service available or not

- Combined service for passenger and freight distribution

- Use of reserved bus lanes

Currently, all the indicated service and operational strategies are not implemented, but they will be in the future. The involved factors in the preliminary design of experiments that have been considered are:

- Global Traffic Demand Scenarios: Testing has been performed in the morning rush-hour scenario, allowing request generation in the first $30 \mathrm{~min}$.

- Demand Request Generation: There are two options for generating requests; either as a percentage of the passenger cars demand (the use OD pattern), or randomly distributed over the area. The first option has been selected and requests are generated over a circle with a ratio of 200 meters around the centroide constrained to a minimum travel distance of $0.5 \mathrm{~km}$ (to be set). Hence the total number of trips for the new mode are set to 5\%,10\% or 15\% of the total passenger cars demand in the study period. Figure 3 shows an example of the distribution over the site of 1000 requests (origins in red and destinations in green).

- Capacity of the units that operate in the new mode. Seated capacities rank are set to 8, but it is a configurable parameter (from 6 to 10 passengers will be assessed in the future).

- Fleet dimension: it was considered between 250, 500, 750 and 1000 vehicles. A no depot solution was implemented and fleet vehicles are parked in specific allowed zones at the beginning/end of the passengers' transport journey, these emulated zones are the regular loading/unloading area for freight distribution in the chamfers across the road network of Eixample. When a new mode's unit is not operating (i.e. it is empty and it is waiting for the arrival of new request), it goes and parks in the allowed zones until a new requested demand has to be served. Fleet is allowed to use bus restricted lanes and also to pick up and deliver passenger in bus-stops.

- Time Windows Width: The width of the time interval in which the demand requests must be served (pickup and delivery time windows). Considered levels are 10,15 and $30 \mathrm{~min}$ and the base for comparison where no restriction is assumed. 


\section{Linares, Montero, Barceló and Carmona}

- Degree of Dynamism: The \% with respect to the total demand for the study period that is not prebooked in advance (\% real time requests). Considered levels are $0 \%, 20 \%, 50 \%, 80 \%$ and $100 \%$. The "Customer Request Emulator" is an API specifically developed to generate three types of new mode demand requests (on-time demand, pre-booked demand to be served when the simulation starts, and pre-booked demand to be served at a certain specific time). The percentage of each of these proportions was defined to be modified in the settings of the system.

The Base scenario for comparison purposes is defined for a $10 \%$ of total passenger car demand, fleet size 500 vehicles, a pre-booked demand of $50 \%$ and the time-window length is $15 \mathrm{~min}$.

Aimsun's result tables account for statistical performance measures for each user-defined interval of time splitting results into vehicle classes. Nevertheless, the new mode has a fleet of vehicles whose routes do not have an origin-destination, but correspond to a dynamic vehicle routing problem optimizing the overall requests using the available fleet size and capacity, real-time traffic conditions and time-window limitations. Thus an adhoc module was designed and implemented to gather all the data from the new mode operations required to calculate the performance measures. The collected results for each simulation replica, fleet unit and simulation step are the position of each fleet vehicle and its number of passengers.

From the passenger's point of view the defined performance indicators are: average travel time per passenger (in-vehicle plus waiting time); average in-vehicle time per passenger; average service speed (effective travel time divided into Manhattan pick-up and delivery distance); average waiting time; rejected requests. From the point of view of the operator: average number of persons per vehicle-unit; average number of occupied vehicles; number of person-trips per hour; average commercial speed per vehicle-unit; total vehicle-km. For each replication, statistical data was stored in the default Aimsun database and the specific Redis DB designed to collect data for multiple ride-sharing service.

\subsection{Analysis of Simulation results}

The computational time strongly depends on the fleet size and the total number of requests, on average computing times per replica has resulted about 10 hours. Previously, a testing on the Base scenario about the variability of selected performance indicators under a $95 \%$ statistical confidence was undertaken. For example, we obtained that a relative precision of less than $10 \%$ in the effective service speed performance indicator would need around 125 served requests per replica (requests are independently generated over time). Each of our replicas contained more than this amount of served requests for the horizon study.
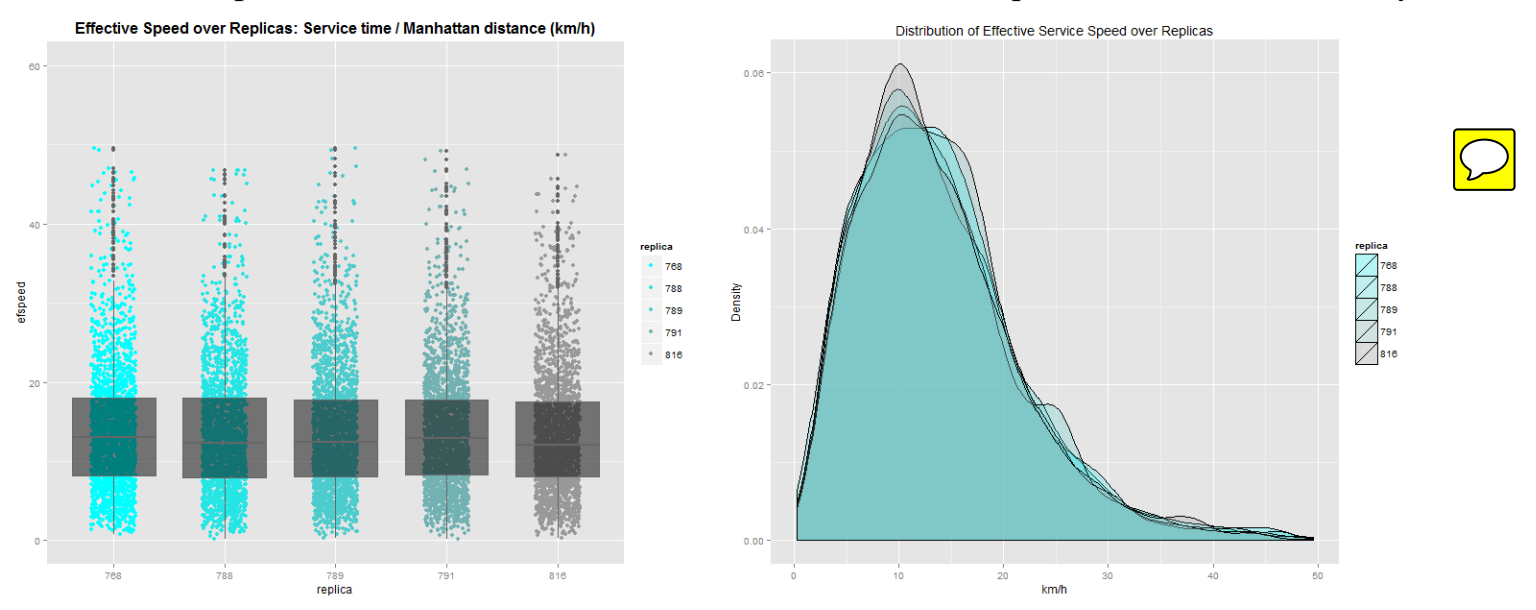

Figure 4: Effective passenger service speed $(\mathrm{km} / \mathrm{h})$ performance indicator in the base scenario: variability study across replicas.

A reasonable number of 5 replicas was considered in the design of experiments since according to the base scenario and selected performance indicators the relative estimation error was less than $5 \%$ at $95 \%$ 


\section{Linares, Montero, Barceló and Carmona}

statistical confidence. For example, the average passenger service speed across replicas was 13.78 min and its variance $0.0449 \mathrm{~min}^{2}$ leading to a relative estimation error less than $2 \%$ or absolute error around $0.25 \mathrm{~km} / \mathrm{h}$ at a $95 \%$ confidence level. Figure 4 shows the effective service speed over replicas, but variability was checked in order to guarantee the quality of the obtained results. A non-parametric Kruskal-Wallis test was undertaken in the Base scenario confirming that the null hypothesis of equal effective service speeds across replicas could not be rejected. Equal variability across replicas for the effective service speed was also accepted. From the point of view of MPRS units and for one replica in the base scenario, results show an average commercial speed for the new mode that depends on the occupancy (see Figure 5). The median of occupancy is 2.0 passenger/veh when non-empty, and commercial speed when 1 to 4 passengers occupied the MPRS unit behaves on the mean with no differences (according to hypothesis testing results), while over 5 passengers speed increases at $95 \%$ confidence level.
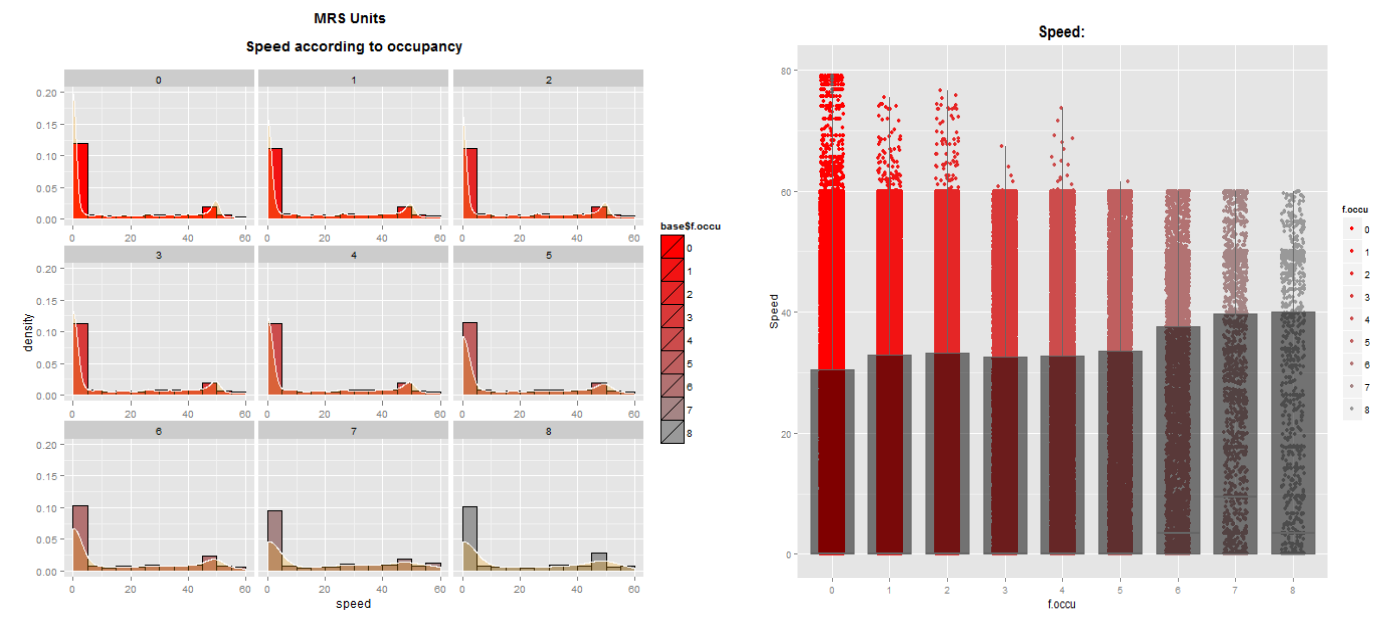

Figure 5: Commercial speed of vehicle units $(\mathrm{km} / \mathrm{h})$ while non-empty according to passenger occupancy (histograms-left and boxplots-right). Base scenario.

A graphic representation of a fixed replica can be obtained postprocessing the adhoc Redis database for fleet collecting data during the simulation. Time window restriction on the pick-up and arrival time was found to be the most important factor in the experiments design. In Figure 6, for the average in-vehicle travel time per passenger, a tridimensional plot considering the most important factors in the design are shown. Clearly, as the percentage of dynamism in the requests decreases, the average in-vehicle time decreases, assuming a pre-booked demand from the previous day or horizon before the current simulation period, the time window factor defines the planes in Figure 6 and show a significant effect that explains nearly $80 \%$ of the total variability of in-vehicle travel time. The coefficient of determination for the linear model of in-vehicle travel time on the percentage of dynamism, the time-window length and the percentage of the total travel demand for the period served by the new mode is almost $85 \%$. Clearly, an infinite time-window (no restriction) contributes to increase in-vehicle travel times for passengers. The fleet size is a non-significant variable to explain the in-vehicle travel time for a passenger.

Fleet size has an important effect for explaining the percentage of served requests according to the statistical results analysis of the linear model for the percentage of the served request on fleet size, $\%$ MPRS demand, \%Pre-booking and Time-Window length variables; fleet size accounts for $80 \%$ of total variability in the response, followed by \%MPRS demand and time-window length. The percentage of prebooked demand has proved to be non-significant.

Figure 7 shows how the percentage of time consumed to pick up customers fast increases as the demand for the new mode increases for a fleet size fixed to 500 units; but, clearly it shows a non-linear relationship with the percentage of served requests, that has to be analyzed in depth in future research. 

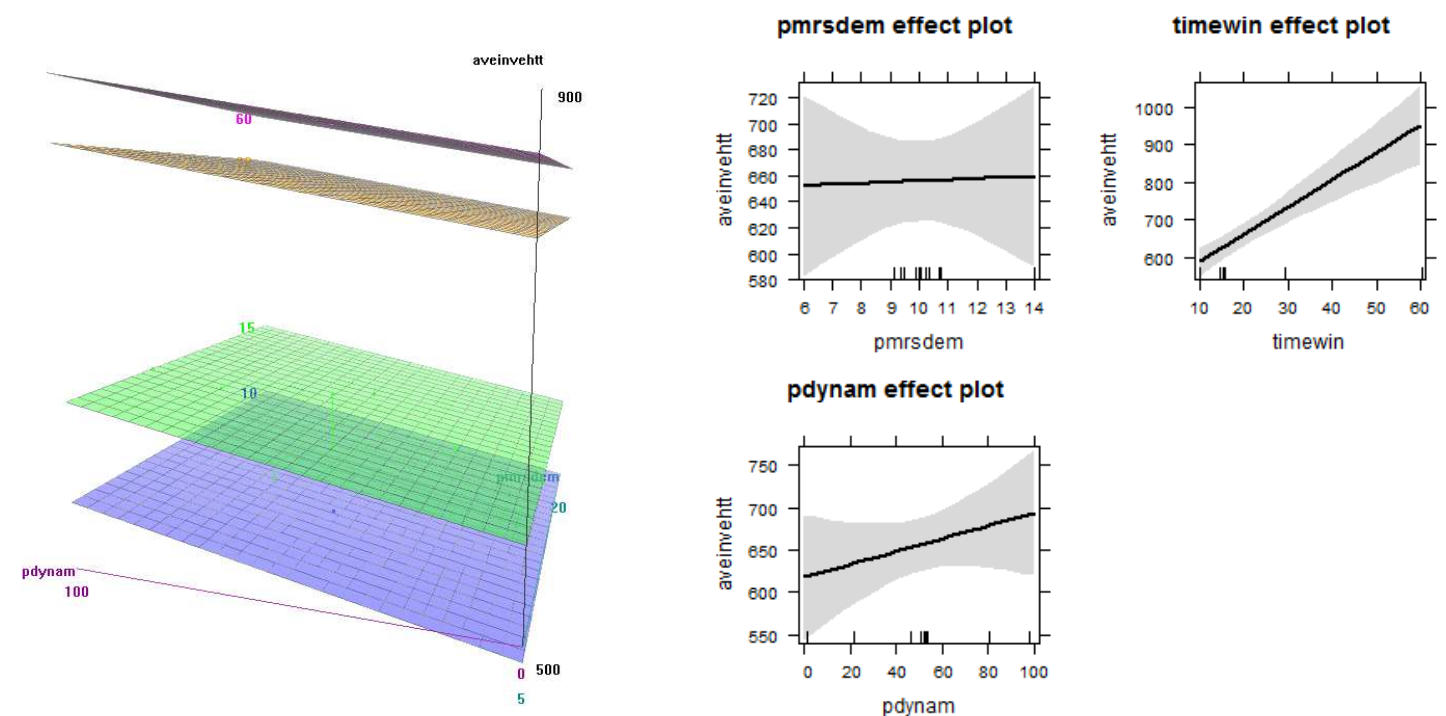

pdynam effect plot

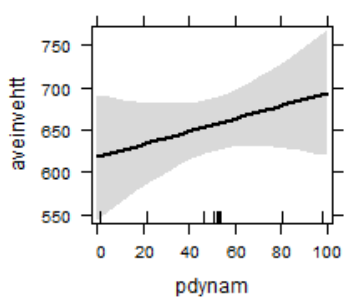

Figure 6: Average in-vehicle travel time (sec) depending on time-window restriction, the percentage of dynamic and total demand for the new mode.
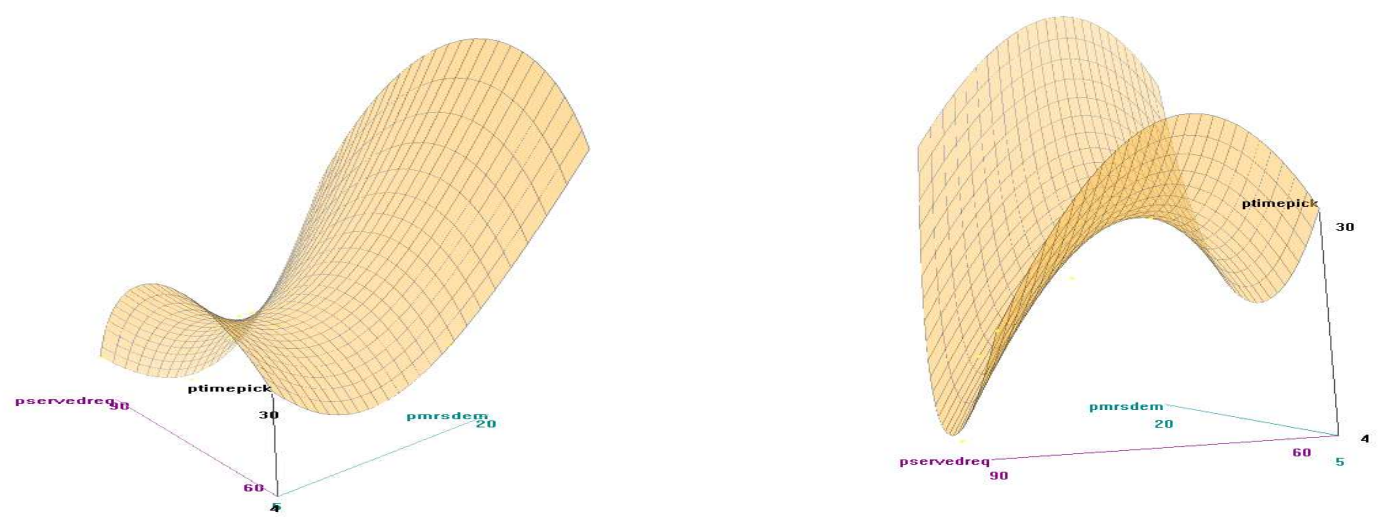

Figure 7 : Percentage of Time consumed to pick up requests depending on the demand for the new mode and the percentage of served requests (two perspectives). Fleet size fixed to 500 units.

\section{CONCLUSIONS AND FUTURE RESEARCH}

The research carried out has been based on an approach consisting on a general framework and simulation architecture to emulate and evaluate general policies of this new mobility services, which has demonstrated the potential feasibility and impacts of some of the proposed mobility concepts for on demand multiple passenger ride-sharing in urban area. In the near future other options will be tested.

\section{ACKNOWLEDGMENTS}

This research was funded by TRA2014-52530-C3-3-P of the Spanish R+D National Programs and by the Secretaria d'Universitats i Recerca de la Generalitat de Catalunya under 2014 SGR 1534. Throughout, the authors have benefited from the support of R.M. Martín, G. Navarro and A. Rodríguez from inLab FIB. 


\section{REFERENCES}

Anon, 2015. KUTSUPLUS. Available at: https://kutsuplus.fi.

Barceló, J., Orozco, J.-A. \& Grzybowska, H., 2013. Making Real-Time Fleet Management Decisions Under Time-Dependent Conditions in Urban Freight Distribution. In E. V. de V. (ed. . Moshe BenAkiva, Hilde Meersman, ed. Freight Transport Modelling. pp. 453-484.

Boesch, M.P., Ciari, F. \& Axhausen, K.W., 2016. Required autonomous vehicle fleet sizes to serve different levels of demand. In Proceedings of the 94th Transportation Research Board Annual Meeting. Washington D.C., p. Paper 16-2682.

Chabini, I., 1998. Discrete Dynamic Shortest Path Problems in Transportation Applications: Complexity and Algorithms with Optimal Run Time. Transportation Research Record: Journal of the Transportation Research Board, 1645, pp.170-175. Available at: http://trrjournalonline.trb.org/doi/abs/10.3141/1645-21.

Fagnant, D. \& Kockelman, K., 2014. The travel and environmental implications of shared autonomous vehicles, using agent-based model scenarios. Transportation Research Part C: Emerging Technologies, 40, p.pp 1-13.

Furuhata, M. et al., 2013. Ridesharing: The state-of-the-art and future directions. Transportation Research Part B: Methodological, 57, pp.28-46.

Ma, S., Zheng, Y. \& Wolfson, O., 2015. Real-time city-scale taxi ridesharing. IEEE TRANSACTIONS ON KNOWLEDGE AND DATA ENGINEERING, 27(7), pp.1782-1795. Available at: http://ieeexplore.ieee.org/xpls/abs_all.jsp?arnumber $=6847170$.

Martinez, L.M., Correia, G.H.A. \& Viegas, J.M., 2015. An agent-based simulation model to assess the impacts of introducing a shared-taxi system: An application to Lisbon (Portugal). Journal of Advanced Transportation, 49(3), pp.475-495.

OECD - International Transport Forum, 2015. Urban Mobility System Upgrade: How shared self-driving cars could change city traffic. Corporate Partnership Board Report, pp.1-36.

Sullivant \& Frost, 2015. Intelligent Mobility 3.0 Future of Mobility \& New Mobility Business Models. In Sullivan and Frost, ed. London. Available at: http://www.frost.com/sublib/displayreport.do?id=MB85-01-00-00-00.

Transport Simulation Systems, 2013. Aimsun 7 Dynamic Simulator User's Manual.

Ziliaskopoulos, A.K. \& Mahmassani, H., 1993. Time-dependent shortest path algorithm for real-time intelligent vehicle highway system applications. Transportation Research Record, 1408, pp.94-100.

\section{AUTHOR BIOGRAPHIES}

MaAZ LINARES is a Ph.D. researcher at the inLab FIB (Barcelona informatics school laboratory) on the area of Mathematical Programming, Logistics and Simulation. Her research concerns dynamic traffic assignment, traffic simulation and optimization. Email: mari.paz.linares@upc.edu.

LÍDIA MONTERO is Associate Professor of the Department of Statistics and Operations Research at UPC. Her research concerns simulation-optimization issues, with applications to transport engineering and demand modeling. Email: lidia.montero@upc.edu.

JAUME BARCELÓ is Professor Emeritus at the department of Statistics and Operations Research at UPC and the head of ICT and Transport Projects at inLab FIB. Email: jaume.barcelo@upc.edu.

CARLOS CARMONA is a researcher at the inLab FIB. He received his computer engineering from the Barcelona School of Informatics (FIB). He works developing projects related to the simulation and optimization of processes since 2002. Email: carlos.carmona@fib.upc.edu. 